\title{
INCIDENCIA DE PATOLOGÍA SALIVAL SINTOMÁTICA ALEJADA EN PACIENTES TRATADOS CON YODO RADIOACTIVO POR CÁNCER DIFERENCIADO DE TIROIDES*
}

\author{
Drs. Ignacio Goñi E. ${ }^{1}$, Guillermo Vander Selt A. ${ }^{1}$, EU. Catalina Ruiz A. ${ }^{1}$, \\ Augusto León R. ${ }^{1}$, Antonieta Solar G. ${ }^{2}$, Pilar Orellana B. ${ }^{3}$ \\ Departamento de Cirugía Oncológica y Maxilofacial, División de Cirugía. \\ 2 Departamento de Anatomía Patológica. \\ 3 Departamento de Radiología y Medicina Nuclear. \\ Pontificia Universidad Católica de Chile. \\ Santiago, Chile.
}

\begin{abstract}
\section{Incidence of sympthomatic salivary disease in patients with differentiated} thyroid cancer treated with radioactive iodine
\end{abstract}

Background: The radioactive iodine therapy for differentiated thyroid cancer can produce severe and frequent salivary symptoms, during the treatment or later. Aim: To analyze the incidence, severity and charactheristics of the salivary signs and symptoms in these patients. Patients and Method: Retrospective and descriptive analisis of 106 patients with confirmed diagnosis of differentiated thyroid cancer, treated with surgery and radioactive iodine, that completed a telephonic survey for the evaluation of salivary symptoms. Results: $26(24.52 \%)$ patients presented with salivary symptoms or signs after the radioactive iodine therapy (mean 5 months). The average doses of I 131 was $128,5 \mathrm{mCi}$. Xerostomy, pain, xeroftalmy, inflammation, sialoadenitis and dysgeusia, were the most frequent clinical symptoms. Conclusions: After radioactive iodine therapy the salivary symptoms and signs incidence is high. We conclude that the indication for this treatment must be selective, but in accordance with the oncological risk of each patient.

Key words: Radioactive iodine therapy, thyroid cancer.

\section{Resumen}

Introducción: El tratamiento con yodo radioactivo en el tratamiento del cáncer diferenciado de tiroides puede originar síntomas alejados de origen salival. Éstos pueden llegar a ser intensos y frecuentes. Objetivo: Conocer la incidencia, características e intensidad de dichos síntomas. Material y Método: Revisión retrospectiva y análisis descriptivo de 106 pacientes con diagnóstico definitivo y anatomopatológico de cáncer diferenciado de tiroides, tratados con yodo radioactivo, que contestaron una encuesta telefónica

*Recibido el 27 de mayo de 2014 y aceptado para publicación el 11 de junio de 2014.

Los autores no refieren conflictos de interés.

Correspondencia: Dr. Ignacio Goñi E.

igoni@med.puc.cl 
especialmente diseñada para evaluación de patología salival. Resultados: Veintiséis (24,52\%) pacientes presentaron y consultaron por síntomas y/o signos alejados (promedio 5 meses) de la terapia ablativa, de origen salival. La dosis promedio fue de 128,5 mCi de I 131. Los síntomas más frecuentes fueron xerostomía, dolor, xeroftalmia, inflamación, sialoadenitis y alteración del gusto. Discusión: La incidencia de signos y síntomas salivales alejados en pacientes tratados con I 131 es alta y justificaría a nuestro juicio su indicación selectiva, de acuerdo a los riesgos de recurrencia tumoral de cada paciente.

Palabras clave: Radioyodo, cáncer de tiroides.

\section{Introducción}

El cáncer diferenciado de la glándula tiroides (CDT) tendría una prevalencia en Chile de $3 \%$ de la población. En el mundo, su incidencia varía entre 0,5 y 10 por 100.000 habitantes y corresponde al cáncer endocrino más frecuente ${ }^{l}$. El tratamiento del CDT consiste en tiroidectomía total o lobectomía tiroídea, con o sin disección ganglionar cervical selectiva, y utilización adyuvante de radioyodo (RAI). Ésta ha sido indicada en la mayoría de los pacientes, utilizándose como herramienta fundamental para el control locorregional microscópico de la enfermedad, la ablación de los restos de glándula tiroides posterior al acto quirúrgico y para lograr utilidad en la medición de la tiroglobulina, como marcador tumoral posterior al tratamiento ${ }^{2}$. Además, la terapia con RAI es eficaz para el control de la enfermedad localmente avanzada o metastásica ${ }^{1,3}$. Clásicamente, la dosis de ablación ha fluctuado entre 75 y 150 $\mathrm{mCi}$. Actualmente en algunos centros, incluido el nuestro, se han ajustado las dosis y la indicación de RAI a cada caso en particular, dependiendo de los factores que en el paciente condicionan mayor riesgo de recurrencia y falla del tratamiento en general ${ }^{3-5}$.

La incidencia de efectos colaterales de la aplicación de RAI utilizado como ablación ha sido publicada en variados estudios, con síntomas de patología salival alejada hasta en un $39 \%$ de los pacientes ${ }^{6-8}$. Los síntomas derivados del efecto del RAI sobre el tejido glandular salival y lagrimal corresponden a xerostomia, dolor y aumento de volumen, sequedad ocular y alteraciones del gusto ${ }^{6-9}$.

La acción del radioyodo sobre el tejido salival ha sido explicada en estudios previos ${ }^{10} \mathrm{y}$ tendría efecto clínico variable de acuerdo a la dosis utilizada y acumulada. Generalmente los efectos sobre el tejido salival son moderados y tienden a disminuir en el tiempo y se presentan principalmente durante el primer año posterior al tratamiento 9 . Los síntomas pueden llegar a hacerse permanentes hasta en el $15 \%$ de los pacientes ${ }^{10-14}$.

\section{Objetivo}

Conocer la incidencia y características de síntomas sugerentes de patología salival alejada en pacientes sometidos a radioyodo, como terapia adyuvante en cáncer diferenciado de tiroides.

\section{Material y Método}

Revisión retrospectiva y análisis descriptivo de la base de datos de los Departamentos de Cirugía Oncológica-Maxilofacial, Anatomía Patológica y de Radiología de la Pontificia Universidad Católica de Chile, de los pacientes operados por cáncer diferenciado de tiroides entre los años 2007 a 2010, que hayan recibido yodo radioactivo terapéutico. Se registraron las dosis de RAI administradas a cada paciente. Se aplicó una encuesta por vía telefónica, en la que se consignaron los datos demográficos, presencia de enfermedades del mesénquima, síntomas durante el tratamiento y en forma alejada, momento de aparición de los síntomas e intensidad de éstos.

Los criterios de inclusión correspondieron a pacientes con certeza anatomopatológica del diagnóstico de cáncer diferenciado de tiroides que recibieron tratamiento adyuvante con RAI y que hayan contestado la encuesta telefónica. Los criterios de exclusión correspondieron a pacientes con enfermedades del mesénquima previo a tratamiento con RAI.

Los datos son presentados en promedios con desviación estándar y moda, cuando correspondía. Comparación de promedios entre grupos con T-test para muestras independientes. El análisis se realizó con programa Stata (versión 11.0).

\section{Resultados}

De un total de 1.175 pacientes operados por cáncer diferenciado de tiroides durante los años 2007 a 2010, se incluyeron 106 pacientes que recibieron adyuvancia con RAI en este período y que accedieron a contestar la encuesta. El promedio de edad fue de 45,38 años (DE 13,06), rango 22 a 78 años. El 91,91\% fue de sexo femenino. Fueron excluidos 5 pacientes por presentar patología mesenquimática previo al tratamiento con RAI, de éstos, tres presentaban artritis reumatoides, uno fibromialgia y uno lupus/síndrome de Sjögren.

Se presentaron síntomas durante el tratamiento 
en $52(49,05 \%)$ pacientes. El 96,07\% fueron mujeres, siendo la edad promedio de 44,27 (DE 12,02) rango 23 a 75 años. El síntoma más frecuente fue alteración del gusto, que se presentó en $36(69,23 \%)$ de los pacientes, seguido por xerostomía en 21 $(40,38 \%)$ pacientes, dolor en $12(23,07 \%)$ y aumento de volumen en $4(7,69 \%)$.

Se presentaron síntomas alejados en 26 (24,52\%) pacientes (Tabla 1), la mitad de los cuales no habían presentado molestias salivales durante el tratamiento. De éstos, un solo paciente fue de sexo masculino y la edad promedio fue de 45,62 (DE 12,91), rango 23 a 78 años. De este grupo, con sintomatología alejada, el síntoma más frecuente fue xerostomía en $14(53,84 \%)$ pacientes, dolor en $11(42,30 \%)$, xeroftalmia en $6(23,07 \%)$, aumento de volumen en $6(23,07 \%)$, sialoadenitis se presentó en $2(7,69 \%)$ y alteración del gusto en 1 paciente.

Las dosis administradas de RAI fluctuaron entre 100 a $200 \mathrm{mCi}$, siendo en promedio 128,24 (DE 34,89), con una moda de $100 \mathrm{mCi}$. La dosis promedio fue de 132,95 (DE 37,26) mCi y 123,17 (DE 31,81$) \mathrm{mCi}$, para el grupo con y sin síntomas durante el tratamiento, respectivamente $(p=0,19)$ y de $137,5 \mathrm{mCi}$ y $124,59 \mathrm{mCi}$, para el grupo con y sin síntomas alejados de tipo salival, respectivamente $(\mathrm{p}=0,125)($ Tablas 2 y 3$)$.

Los síntomas durante el tratamiento y alejados se presentaron en un $46,5 \%$ y $23,4 \%$ para el grupo que recibió $100 \mathrm{mCi}, 53,5 \%$ y 28,27\% para el grupo que recibió $150 \mathrm{mCi}, 70$ y $50 \%$ para el grupo que recibió $200 \mathrm{mCi}$.

El promedio de seguimiento fue 5 meses, con una moda de 4 meses en rango de 1-12, destacando que $8(7,54 \%)$ pacientes continuaron con síntomas por más de 12 meses. La severidad de los síntomas referida en una escala de 1 a 10, siendo 1 muy leve y 10 muy intensos, fue en promedio de 5,48 (DE 2,48) en rango de 2 a 10 , con una moda de 4 . No existieron diferencias estadísticamente significativas con respecto a la dosis de RAI y la presencia de síntomas durante el tratamiento o síntomas alejados.

Tabla 1. Pacientes con síntomas salivales durante y alejado del tratamiento

\begin{tabular}{|lccc|}
\hline $\begin{array}{c}\text { Total de pacientes } \\
\mathbf{n} / \mathbf{\%}\end{array}$ & $\begin{array}{c}\text { Con síntomas durante } \\
\text { el tratamiento } \\
\mathbf{n} / \mathbf{\%}\end{array}$ & $\begin{array}{c}\text { Con síntomas alejados del } \\
\text { tratamiento } \\
\mathbf{n} / \mathbf{\%}\end{array}$ \\
\hline Edad promedio & $106 / 100$ & $52 / 49,05$ & $26 / 24,52$ \\
\hline
\end{tabular}

Tabla 2. Datos comparativos en relación a la dosis de I ${ }^{131}$ administrada. Grupos con o sin síntomas durante el tratamiento

\begin{tabular}{|cccc|}
\hline Dosis I ${ }^{131}(\mathbf{m C i})$ & Máxima & Mínima & Promedio \\
\hline Sin síntomas & 200 & 100 & 123,17 \\
Con síntomas & 200 & 100 & 132,95 \\
& & & $\mathrm{p}=0,19$ \\
\hline
\end{tabular}

Tabla 3. Datos comparativos en relación a la dosis de I ${ }^{131}$ administrada. Grupos con o sin síntomas alejados del tratamiento

\begin{tabular}{|cccc|}
\hline Dosis I ${ }^{131}(\mathbf{m C i})$ & Máxima & Mínima & Promedio \\
Sin síntomas & 200 & 100 & 124,59 \\
Con síntomas & 200 & 100 & 137,5 \\
& & & $\mathrm{p}=0,125$ \\
\hline
\end{tabular}




\section{Discusión}

La terapia ablativa con yodo radioactivo se ha considerado un tratamiento adyuvante importante en el manejo de los pacientes con diagnóstico de cáncer diferenciado de tiroides. Su utilización persigue básicamente eliminar por medio de radiación interna tanto el tejido tiró́deo remanente como el eventual tejido tumoral residual local y regional (metástasis linfonodales subclínicas).

Su indicación ha sido establecida basándose en la capacidad de captación de yodo por la célula tumoral diferenciada. Las dosis utilizadas del radioisótopo I 131 varían ampliamente y se ha establecido con criterios de necesidad y seguridad del paciente. Hasta hace unos años se utilizaban rutinariamente dosis del orden de los 75 a $200 \mathrm{mCi}$. Actualmente esta dosis se ha ajustado en valores más bajos, especialmente en pacientes con bajo riesgo de recidiva ${ }^{11,12}$.

La asociación de patología salival con la dosis administrada de RAI se ha demostrado en múltiples estudios ${ }^{9,14}$. En nuestro estudio se presenta una tendencia similar, sin poder obtener datos estadísticamente significativos que apoyen esta asociación.

Los síntomas alejados de origen salival, derivados del uso de este tratamiento, son descritos hasta en el $39 \%$ de los casos ${ }^{8}$. Nuestra cifra fue menor. Probablemente la forma de medir la incidencia de dichos síntomas, determina este valor. En nuestro estudio la utilización de una encuesta telefónica podría incidir en este aspecto, ya que no se realizó un control clínico directo de los pacientes.

De acuerdo a estos resultados, prácticamente 1 de cada 4 pacientes presentó molestias alejadas de tipo salival, sin definirse, dado el diseño del estudio, la eventual cronicidad de ellos. Solans ${ }^{9}$ y Grewal ${ }^{8}$, señalan que en un 5 a $7 \%$ de los casos las molestias persistirán. Esto concuerda con nuestras cifras, donde el 7,5\% de los pacientes persistieron con síntomas por más de 1 año.

Con una serie adecuada en número, que permita el control médico estricto y objetivo, podría analizarse la relación dosis-severidad de los síntomas. Ésta ha sido estudiada en otros estudios con resultados dispares ${ }^{6,8,9}$. Rosario ${ }^{15}$, propone que la preparación para la terapia con RAI con hormona estimulante de la tiroides recombinante humana (rhTSH) se asociaría a una disminución en los síntomas de las glándulas salivales.

En conclusión, el radioyodo seguirá siendo una modalidad de tratamiento importante en el manejo del cáncer de tiroides. La incidencia de síntomas y signos secundarios a patología salival por uso de radioyodo es alta y justifica una indicación selectiva, basándose principalmente en el riesgo de recurrencia tumoral evaluado en cada paciente, intentando maxi- mizar los beneficios, minimizando los potenciales efectos adversos.

\section{Referencias}

1. Landis SH, Murray T, Bolden S, Wingo PA. Cancer statistics, 1998 [published errata appear in CA Cancer J Clin. 1998;48:192 and 1998;48:329].

2. Mazzaferri EL. An overview of the management of papillary and follicular thyroid carcinoma. Thyroid 1999;9:421-7.

3. Mazzaferri EL, Kloos RT. Clinical review 128: current approaches to primary therapy for papillary and follicular thyroid cancer. J Clin Endocrinol Metab. 2001;86:1447-63.

4. DeGroot LJ, Reilly M. Comparison of 30- and 50-mCi doses of iodine-131 for thyroid ablation. Ann Intern Med. 1982;96:51-3.

5. Mercante G1, Frasoldati A, Pedroni C, Formisano D, Renna L, Piana S. Prognostic factors affecting neck lymph node recurrence and distant metastasis in papillary microcarcinoma of the thyroid: results of a study in 445 patients. Thyroid 2009;19:707-16.

6. Samaan NA, Schultz PN, Hickey RC, Goepfer H, Hayne $\mathrm{T}$, Johnston DA, et al. The results of various modalities of treatment of well differentiated thyroid carcinomas: a retrospective review of 1599 patients. J Clin Endocrinol Metab. 1992;75:714-20.

7. Markitziu A, Lustmann J, Uzieli B, Krausz Y, Chisin R. Salivary and lacrimal gland involvement in a patient who had undergone a thyroidectomy and was treated with radioiodine for thyroid cancer. Oral Surg Oral Med Oral Pathol. 1993;75:318-22.

8. Grewal RK, Larson SM, Pentlow CE, Pentlow KS, Gonen M, Qualey R, et al. Salivary Gland Side Effects Commonly Develop Several Weeks After Initial Radioactive Iodine Ablation. J Nucl Med. 2009;50:1605-10.

9. Solans R, Bosch JA, Galofre Ṕ, Porta F, Rosello' J, O'Callagan A, et al. Salivary and Lacrimal Gland Dysfunction (Sicca Syndrome) after Radioiodine Therapy. The Journal of Nuclear Medicine 2001;42;738-43.

10. Cavalieri RR. Iodine metabolism and thyroid physiology: current concepts. Thyroid 1997;7:177-81.

11. Kruijff S, Aniss AM, Chen P, Sidhu SB, Delbridge LW, Robinson B. Decreasing the dose of radioiodine for remnant ablation does not increase structural recurrence rates in papillary thyroid carcinoma. Surgery 2013;154:1337-44; discussion 1344-5.

12. Nixon IJ1, Ganly I, Patel SG, Palmer FL, Di Lorenzo MM, Grewal RK. The results of selective use of radioactive iodine on survival and on recurrence in the management of papillary thyroid cancer, based on Memorial Sloan-Kettering Cancer Center risk group stratification. Thyroid 2013;23:683-94.

13. Durante C, Haddy N, Baudin E, Leboulleux S, Hartl D, Travagli JP, et al. Long-term outcome of 444 patients 
with distant metastases from papillary and follicular thyroid carcinoma: benefits and limits of radioiodine therapy. J Clin Endocrinol Metab. 2006;91:2892-9.

14. Hyer S, Kong A, Pratt B, Harmer C. Salivary gland toxicity after radioiodine therapy for thyroid cancer. Clin
Oncol. (R Coll Radiol). 2007;19:83-6.

15. Rosario PW, Borges MA, Purisch S. Preparation with recombinant human thyroid-stimulating hormone for thyroid remnant ablation with $131 \mathrm{I}$ is associated with lowered radiotoxicity. J Nucl Med. 2008;49:1776-82. 